\title{
EFFECTS OF VITAMIN D SUPPLEMENTATION ON WELL-BEING, POSTURAL CONTROL, MUSCLE STRENGTH, BONE AND CALCITROPIC HORMONES - A RANDOMIZED DOUBLE- BLIND PLACEBO CONTROLLED TRIAL
}

\author{
S.A. Eriksen ${ }^{1}$, J. Starup-Linde ${ }^{2}$, R.P. Hirata ${ }^{3}$, K.K. Petersen ${ }^{4}$, T. Graven-Nielsen ${ }^{4}$, P. Vestergaard ${ }^{1,5}$
}

\begin{abstract}
Background: Antidepressants may increase the risk of fractures through negative effects on the musculoskeletal system that could be hindered by vitamin D supplements. Objectives: To study the pleiotropic effects of daily vitamin D supplementation in depressed patients treated with citalopram (patients) and healthy controls. Design: Randomised double blind placebo controlled trial. Setting: A study of Danish women in the age 50 to 90 years. Participants: A total of 21 patients and 50 controls. Intervention: Participants received daily vitamin D supplementation (50 micrograms) or placebo in one year. Measurements: Bone Densitometry by dual-energy x-ray absorptiometry. Serum 25-hydroxyvitamin D, intact-Parathyroid Hormone, type 1 procollagen N terminal peptide, tartrate resistant acid phosphatase type $5 \mathrm{~b}$. Pain sensitivity measures based on pain detection thresholds by cuff algometry, temporal summation of pain, conditioned pain modulation, and cutaneous pain sensitivity by pinprick test. Degree of depression by the Major Depression Inventory. Physical performance was assessed by Timed up and go, isomeric handgrip exercise, and postural control by force plate. Results: Serum 25(OH)D levels increased in the vitamin D treated patients compared with controls at the 12 months visit $(\mathrm{P}<0.05)$. Conversely, intact- Parathyroid Hormone decreased among the patients and controls receiving vitamin $\mathrm{D}$ compared with placebo $(\mathrm{P}<0.05)$. Vitamin $\mathrm{D}$ improved Major Depression Inventory scores in patients and controls compared with placebo $(\mathrm{P}<0.05)$. In patients receiving vitamin $\mathrm{D}$, handgrip strength improved $(\mathrm{P}<0.05)$. Conclusions: Vitamin D may improve depressive symptoms, and improve handgrip strength among patients compared to controls.
\end{abstract}

Key words: Vitamin D, citalopram, BMD, pain sensitivity, physical performance.

\section{Introduction}

Several studies have shown that vitamin D supplementation have a positive influence on Bone Mineral Density (BMD). Higher doses (700 to $800 \mathrm{IU}$ a day) of vitamin D supplementation seemed to be more effective in fracture prevention and prevention of falls than smaller doses (400 to 600 IU a day) among middleaged and older adults (1).

Vitamin D may also affect skeletal muscle cell contractility, differentiation, and proliferation (2), although the effects remain controversial. The synthesis of vitamin $\mathrm{D}$ in the skin decreases with age, as well as absorption in the intestines as well as activation in the

1. Department of Clinical Medicine, Aalborg University Hospital, Aalborg, Denmark; 2. Department of Endocrinology and Internal Medicine, Aarhus University Hospital, Aarhus, Denmark; 3. SMI, Department of Health Science and Technology, Aalborg University, Aalborg, Denmark; 4. Center for Neuroplasticity and Pain (CNAP), SMI, Department of Health Science and Technology, Aalborg University, Aalborg, Denmark; 5. Department of Endocrinology, Aalborg University Hospital, Aalborg, Denmark

Corresponding Author: Stine Aistrup Eriksen, PhD cand.scient.med, Department of Clinical Medicine, Aalborg University Hospital, Aalborg, Denmark, stineaistrup@ hotmail.com, Phone: +4523986072 kidneys and peripheral tissues is lower among elderly people. Further, the level and capacity of the vitamin D receptor is lower with advancing age, with a resultant higher prevalence of vitamin D deficiency within this population. Especially among elderly people, vitamin D deficiency has been associated with an important determinant of disability, comprising poor physical performance, muscle fatigue, falls and fractures (3). Falls and fractures are likewise strongly associated with muscle weakness, gait and balance deficits (4), as well as presence of musculoskeletal pain (5).

Major depressive disorder (MDD) is prevalent especially in the geriatric population. Medical treatment with antidepressants have significantly advanced the outcome of depression although a number of remitted depressed persons still experience residual symptoms like fatigue, myalgia, and bone pain (6). These symptoms resemble those seen in patients deficient in vitamin $D$ and recent meta-analyses have reported evidence for an association between hypovitaminosis D and MDD (7). Low exposure to sunlight because of isolation at home and poor dietary habits may be consequences of the depressive condition, affecting vitamin D levels 
negatively.

Chronic pain is common in MDD where approximately $70 \%$ of patients with MDD may experience chronic pain (8). The association is most likely bidirectional since depression predict persistent pain and pain predict depressive relapse and persistence, as prognosis and treatment outcome of depression are affected negatively by the co-existence of pain (9). Antidepressants are known to have analgesic properties and have, in addition to treatment of depressive symptoms, been prescribed in the treatment for pain. Specifically patients with impaired pain inhibitory pathways have been shown to achieve greater pain efficacy to duloxetine, a serotoninnoradrenalin reuptake inhibitory, compared to patients with less impaired pain inhibitory pathways (10), suggesting common pathways between depression and chronic pain.

A recent meta-analysis showed that vitamin $\mathrm{D}$, among other vitamins, comprises a potential for reducing depressive symptoms when supplemented in adjunction to antidepressant treatment (11). Another meta-analysis (7) found evidence for an association between 25(OH)D levels and depressive symptoms. Such effects are likely mediated through the recently discovered involvement of vitamin D in regulation of neurotransmitters such as serotonin and noradrenaline (12), which are seen as central components of MDD pathophysiology, and are the main targets of antidepressants. Vitamin D deficiency has also been found to be associated with increased pressure pain sensitivity (13), but the results of treatment with vitamin $\mathrm{D}$ on pain varies $(14,15)$.

Selective serotonin reuptake inhibitors (SSRIs) are often prescribed as first line treatment for MDD in elderly people due to its favourable side-effect profile compared to other types. Recently, SSRIs have however been associated with decreased bone mineral density (BMD) (16) and an increased risk of bone fractures (17).

The aim of this study was in a randomized placebo controlled setting to evaluate the effects of 50 micrograms of daily vitamin $\mathrm{D}$ in patients treated with citalopram for major depressive disorder as well as healthy controls on: 1) General well-being expressed as major depression inventory (MDI) score and EQ-5D as a measure of quality of life. 2) Symptoms associated with depression and lack of well-being such as pain and pain sensitivity. 3) Muscle function and strength expressed as postural control measured by force plate and handgrip strength. 4) The more classical vitamin D on bone and bone turnover as well as calcitropic hormones.

\section{Methods}

The study consisted of a randomised double blind controlled trial of 50 micrograms ( $\mu \mathrm{g})$ (2000 IU) cholecalciferol (vitamin D3) per day versus matching placebo for 12 months. The study was approved by the local ethics committee (N-20130052) and registered on clinical trials.gov (NCT01932931). The study followed the Helsinki declaration and all participants gave informed written consent.

\section{Subjects}

Subjects were recruited between March 2014 and February 2015 through local newspaper advertisement, local hospitals, and pharmacies as well as advertisement on social media.

Inclusion criteria were women aged 50 to 90 years, who were current citalopram or mirtazapine users, or individuals who were going to initiate treatment of either drug within the following two months due to a diagnosis of major depressive disorder; or healthy controls, i.e. not depressed or receiving antidepressants. Due to few participants $(n=3)$ in the Mirtazapine group as originally planned, results from this group will not be presented. The exclusion criteria were: 1 ) Current or use within the past 6 months of drugs affecting bone turnover such as corticosteroids, hormone replacement therapy in postmenopausal women, drugs against osteoporosis or other bone diseases (Paget's disease of bone), vitamin D supplementation $>35$ micrograms daily, depot Medroxyprogesterone Acetate (DMPA), Cyclosporine (CsA), Antiretroviral Therapy (ART). 2) Impaired renal function (serum creatinine $>150$ micromolar/1). 3) Pregnant women. 4) Individuals diagnosed with cancer or a metabolic disorder such as diabetes. 5) Individuals with prosthetic material in hip or spine. 6) Individuals diagnosed with a disease that affects bone such as Paget's disease of the bone, or fibrous dysplasia. 7) Individuals which is not considered eligible for the clinical trial e.g. individuals diagnosed with dementia, severely psychotic or depressed individuals. 8) Individuals that have been taking other antidepressants than citalopram or mirtazapine for more than 6 months prior to the inclusion and if this treatment persisted for a period of minimum 12 months. 9) Individuals who cannot stand up and stand still without support or a helping device due to physically impairment. 10) The presence of other pain problems (e.g. osteoarthritis) or sensory dysfunction (e.g. fibromyalgia, neuropathic pain).

\section{Bone mineral density}

Bone Densitometry (BMD) of total hip, femoral neck, and lumbar spine (L1-L4) was assessed for all subjects at baseline and after 12 months using by dual-energy x-ray absorptiometry (DXA) scans on a GE Lunar Prodigy Scanner (GE Healthcare Lunar prodigy, USA). Quality control procedures were run every day including calibration by use of QA Phantom (Lunar DPX Series QC Phantom and a Block Phantom). The coefficient of variation $(\mathrm{CV})$ between the two scanners was $1 \%$. 


\section{Biochemical measures}

Samples were immediately frozen at $-80^{\circ} \mathrm{C}$. Serum vitamin D [total serum 25-hydroxyvitamin D $(25(\mathrm{OH})$ D)] was measured using an electrochemiluminescencebinding assay ECLIA (Elecsys ${ }^{\circledR}$ Vitamin D total, modular analytics E170). CV was 1.7-7.8 \% for intra-assay and CV 2.2-10.7\% for inter-assay variation.

Procollagen type $1 \mathrm{~N}$-terminal peptide (P1NP), Tartrate-resistant phosphatase type $5 b$ (TRAP5b - iSYS), and intact PTH were determined using an iSys machine and kits from IDS plc. Intact PTH was measured using the IS3200 and control kit (detection limit $5 \mathrm{pg} / \mathrm{ml}$, CV 4.18.2\%). P1NP was measured using the IS4000 and control kit (detection limit $2 \mathrm{ng} / \mathrm{ml}, \mathrm{CV} 4,4-5.3 \%$ ). TRAP5b was measured using the IS4100 and control kit (detection limit $0.9 \mathrm{IU} / \mathrm{l}, \mathrm{CV} 5.0-13.6 \%$ ).

\section{Self-rated questionnaires}

All subjects filled out questionnaires at each visit at the clinic including information regarding mental and physical health status: Major Depression Inventory (MDI) (18), and EQ-5D (19).

\section{Postural control}

Subjects were tested during quiet bipedal stance on a force platform (Plux Biosignals S.A, Arruda dos Vinhos, Portugal) in four different sensory conditions: i) eyes open, standing on a firm surface, ii) eyes closed on firm surface, iii) eyes open on soft surface, iv) eyes closed on soft surface, each lasting for 35 seconds. One trial consisted of the four sensory conditions i) to iv) recorded in the same order and in one sequence, repeated three times separated by short breaks (30-60 seconds) in between. For the soft surface condition, a soft foam pillow (O'live Balance pad, Denmark) was placed on top of the force platform. Average parameters from the three trials, for each of the four sensory conditions, were used for analysis. The center of pressure (CoP) position was estimated from the vertical force data extracted from the force plate. Coefficient of variation (CV) for the four different conditions was determined in a pilot study of seven healthy adults (aged 24-31years) and was $<8.1 \%$ for both CoP range and velocity in the anterior-posterior and medial-lateral direction.

\section{Timed up and go test (TUG)}

The subject started from a sitting position in an arm chair (seat height approximately $43-47 \mathrm{~cm}$ ) and were asked to rise from the chair on an "three, two, one, go" start-signal and walk three meters (marked on the floor), and walk back to the chair and sit down again. Time was recorded manually from the "go" signal and stopped when the subject again was sitting in the chair. The test was repeated three times and the best (fastest) selected for further analysis. CV was calculated in a pilot study on healthy adults $(n=8)$, aged $24-53$ years, and was $3.7 \%$.

\section{Handgrip strength}

Isometric handgrip strength was measured using a hand dynamometer (NC70144, Procare.dk, Denmark). Each subject was instructed to perform maximal contraction force with their dominant hand (defined as their "writing hand"), in a seated upright position and the test hand pointing downwards, parallel with the trunk and unsupported. The test was repeated three times. The maximal strength of the three trials was used for further analysis. CV for handgrip strength was $2.9 \%$ in a pilot study of healthy adults $(n=6)$ aged $17-55$ years.

\section{Cutaneous pinprick sensitivity}

The pinprick test is conducted by use of eight metallic weight calibrated pins, flat contact area, diameter of $0.6 \mathrm{~mm}$, with fixed stimulus intensities (weights: 0.8, 1.6, 3.2, 6.4, 12.8, 25.6 and $60.0 \mathrm{~g}$, Aalborg University, Denmark). The test was conducted in a single point on the skin above the left upper trapezius muscle. Pinprick score was defined as the lightest weighted needle, which consistently elicits a sharp or pricking sensation. The average of the three pinprick scores was used in further analyses.

\section{Computer controlled cuff pressure algometry}

Pressure-induced pain cuff stimulation was induced using computerised cuff algometry (NociTech, Denmark, and Aalborg University, Denmark). Zero $\mathrm{cm}$ on the VAS was defined as "no pain" and $10 \mathrm{~cm}$ as "maximal pain". Three different experimental setups were conducted in the following order: Pressure pain detection and tolerance thresholds, temporal summation of pain (TSP), and conditioning pain modulation (CPM).

One cuff was placed around the left lower leg at the belly of the $\mathrm{m}$. gastrocnemius and inflated to a maximum pressure limit of $100 \mathrm{kPa}$. During cuff inflation, the subjects were instructed to continuously rate their pressure-induced pain intensity on the VAS, from the time at which the pressure was perceived as painful, and pressing the handheld button when the pain became intolerable, defined as the cuff Pressure Pain Tolerance Threshold (cuff PTT). If the subject did not reach their pain tolerance level before the maximum stimulation intensity, $100 \mathrm{kPa}$ was used as an estimate for the cuff PTT value. The pressure pain detection threshold (cPDT) was defined as the pressure at which the VAS score exceeded $1 \mathrm{~cm}[20, \mathrm{~s}$.$] . Cuff stimulation was repeated$ three times, separated by a two-minute resting interval and the mean cuff PDT and PTT were used in further 
calculation.

TSP was evaluated by 10 sequential stimuli delivered at $0.5 \mathrm{~Hz}$ at the intensity of $75 \%$ of PTT. Pain intensity was rated continuously on the VAS during the sequential stimuli, and a total of 10 VAS scores were extracted. The test was repeated twice separated by a minimum oneminute break, and the 10 VAS score means were used in the analysis. For analysis of TSP, the mean VAS score was calculated from the first to the 4th stimulus (VAS-I) and from the 7th to the 10th stimulus (VAS-II). The TSP-effect was defined as the difference between VAS-I and VAS-II (i.e. VAS-II minus VAS-I) [21].

CPM was assessed by applying a conditioned stimulus followed by a test stimulus. The conditioning stimulation was applied through a computer-controlled cuff stimulation, where a constant pressure $30 \mathrm{kPa}$ with one tourniquet cuff placed around the belly of the $\mathrm{m}$. gastrocnemius on the contralateral lower leg (right side) acted as a conditioning stimulus. During the cuff test stimulus, cuff PDT and cuff PTT were reassessed. PDT was extracted and the CPM effect was calculated as the difference between the unconditioned and the conditioned test stimulus (i.e. PDTconditioned minus PDTunconditioned).

\section{Power calculation}

With a one-year change in BMD of $2 \%$ and a SD of $2 \%$, 21 patients were desirable in each group (42 in total) with a risk of $5 \%$ for type 1 error and $10 \%$ for type 2 error. Due to potential drop outs, the number was set at 50 ( 25 in the active and 25 in the placebo group).

Primary outcome measures were plasma vitamin D, BMD by DXA of the lumbar spine, femoral neck, and total hip.

\section{Statistical analyses}

Results were reported as means \pm standard error of the mean (SEM). Differences in baseline characteristics comparing citalopram users and controls and vitamin $\mathrm{D}$ treatment versus placebo treatment were tested using t-tests for independent samples, Fisher's Exact test and Chi-Square Test for Independence as appropriate. To test for significant changes in major outcome variables (BMD and biochemistry) among groups (patients/controls) and according to treatment (vitamin $\mathrm{D} /$ placebo) during the 12 months follow-up period, a three-way mixed repeatedmeasures analysis of variance (ANOVA) (time $x$ group $x$ treatment) was used, controlling for both age and BMI. All post-hoc tests were adjusted for multiple comparisons (Bonferroni). Pearson's coefficients was used to examine correlations between the 12 months difference in $25(\mathrm{OH})$ $\mathrm{D}$ and the major covariates (e.g. citalopram dose or MDI score). All analyses were performed using SPSS Statistics for Windows, version 24.0 (Armonk, NY: IBM Corp).

\section{Results}

A total of 21 patients treated with citalopram and 50 controls met the inclusion criteria and completed all baseline measures (Fig. 1). No serious adverse events were recorded during the trial in neither the vitamin $\mathrm{D}$, nor the placebo group.

Figure 1

Flow-chart of inclusion

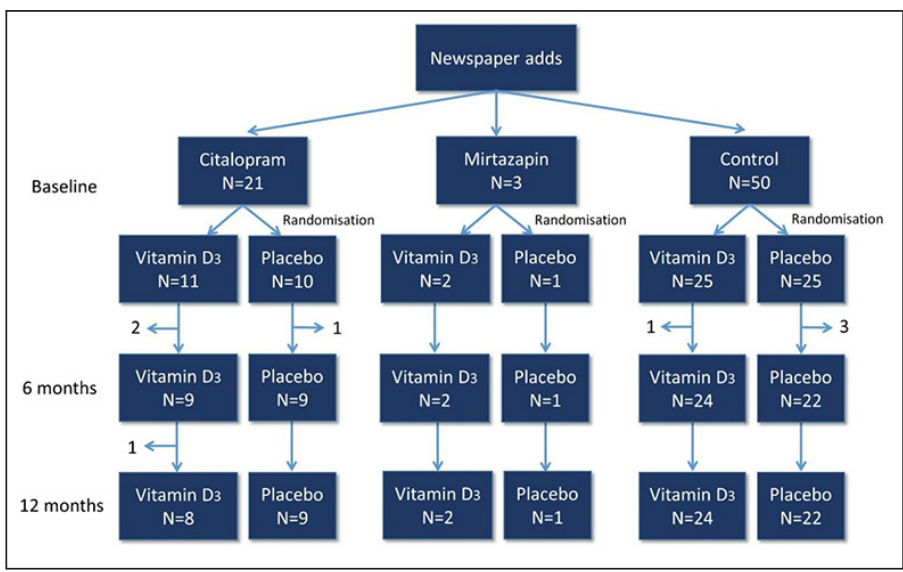

\section{Baseline characteristics}

Table 1 shows baseline characteristics. Average age and systolic blood pressure was higher among patients compared to controls ( $62.6 \pm 1.6$ versus $57.1 \pm 0.8$ years, $\mathrm{P}=0.001)$ and $(139.6 \pm 4.2$ versus $126.4 \pm 2.0 \mathrm{mmHg}$, $\mathrm{P}=0.009)$, respectively. Moreover, alcohol consumption per month was borderline higher among patients compared to controls ( $43 \pm 7$ versus $28 \pm 3$ units/month, $\mathrm{P}=0.054)$. There was no difference in the amount of vitamin D or calcium supplement use at inclusion (normal daily use) at baseline within or between the groups.

\section{Serum Vitamin D}

Figure 2 shows the calcitropic hormones. Serum $25(\mathrm{OH}) \mathrm{D}$ levels increased more among the vitamin $\mathrm{D}$ treated than among the placebo treated for both the citalopram group and the control group. The increase in serum $25(\mathrm{OH}) \mathrm{D}$ levels was higher in the vitamin $\mathrm{D}$ treated citalopram users $(+57.3 \pm 14.5 \mathrm{nmol} / 1)$ than among the controls $(+36.0 \pm 4.3 \mathrm{nmol} / \mathrm{l}, \mathrm{p}=0.03)$, Fig. 2 .

A three-way mixed repeated-measures ANOVA with time (baseline, 6 and 12 months follow-up) as withinsubject factors and group (patient and control) and treatment (vitamin D and placebo) as between-subject factors, adjusting for age and BMI, showed a significant main effect of treatment on serum 25(OH)D levels after 12 months: $\mathrm{F}(2,114) 44.86, \mathrm{p}<0.001, \eta \mathrm{p} 2=0.44$.

iPTH decreased more with vitamin D treatment after 
Table 1

Baseline characteristics. Mean and SEM or proportion

\begin{tabular}{|c|c|c|c|c|c|c|c|}
\hline & & Patients & & & Controls & & Patients vs controls \\
\hline & Vitamin D & Placebo & p-value & Vitamin D & Placebo & p-value & p-value \\
\hline Age (years) & $62 \pm 3$ & $63 \pm 1$ & 0.82 & $57.8 \pm 1.2$ & $56.5 \pm 0.9$ & 0.40 & $<0.01$ \\
\hline Body mass index $(\mathrm{kg} / \mathrm{cm} 2)$ & $27.9 \pm 1.6$ & $26.5 \pm 1.9$ & 0.56 & $25.7 \pm 0.9$ & $25.4 \pm 0.9$ & 0.81 & 0.18 \\
\hline Systolic BP (mmHg) & $144 \pm 6$ & $134 \pm 6$ & 0.32 & $126 \pm 3$ & $127 \pm 3$ & 0.94 & $<0.01$ \\
\hline Diastolic BP (mmHg) & $86 \pm 3$ & $80 \pm 5$ & 0.35 & $82 \pm 2$ & $80 \pm 2$ & 0.37 & 0.46 \\
\hline Pulse (beats / min) & $70 \pm 4$ & $68 \pm 3$ & 0.86 & $68 \pm 1$ & $67 \pm 2$ & 0.75 & 0.41 \\
\hline Citalopram dose (mg/day) & $19.6 \pm 2.4$ & $17.5 \pm 2.5$ & 0.48 & - & & - & - \\
\hline Citalopram duration prior to inclusion (months) & $104 \pm 31$ & $135 \pm 42$ & 0.55 & - & & - & - \\
\hline Never smoking & $7 / 11$ & $4 / 10$ & 0.40 & $12 / 25$ & $12 / 25$ & $1.00^{*}$ & $0.74^{*}$ \\
\hline Years of smoking & $12 \pm 5$ & $21 \pm 8$ & 0.34 & $11 \pm 3$ & $17 \pm 4$ & 0.67 & 0.43 \\
\hline Alcohol consumption (units/month) & $52 \pm 10$ & $33 \pm 10$ & 0.18 & $30 \pm 5$ & $26 \pm 4$ & 0.59 & 0.05 \\
\hline Vitamin D supplements ( $\mu \mathrm{g} /$ day) & $13 \pm 5$ & $19 \pm 7$ & 0.49 & $18 \pm 4$ & $19 \pm 4$ & 0.89 & 0.66 \\
\hline Calcium supplements (mg/day) & $96 \pm 72$ & $106 \pm 60$ & 0.92 & $216 \pm 67$ & $233 \pm 65$ & 0.86 & 0.06 \\
\hline
\end{tabular}

- Fisher's exact test, * Chi square test

12 month among the citalopram treated (change during 12 months $-8.4 \pm 6.1 \mathrm{pg} / \mathrm{ml}$ with vitamin $\mathrm{D}$ vs. $+6.6 \pm 5.5$ $\mathrm{pg} / \mathrm{ml}$ with placebo) than among the controls $(-4.2 \pm 2.3 \mathrm{vs}$. $+0.8 \pm 2.3 \mathrm{pg} / \mathrm{ml}, \mathrm{p}<0.01)$, Fig. 2 .

Figure 2

Absolute changes in calcitropic hormones (25-OH-vitamin $\mathrm{D}$ and PTH) from baseline to 12 months among citalopram treated and controls stratified by treatment group (vitamin D $50 \mu \mathrm{g}$ or placebo)

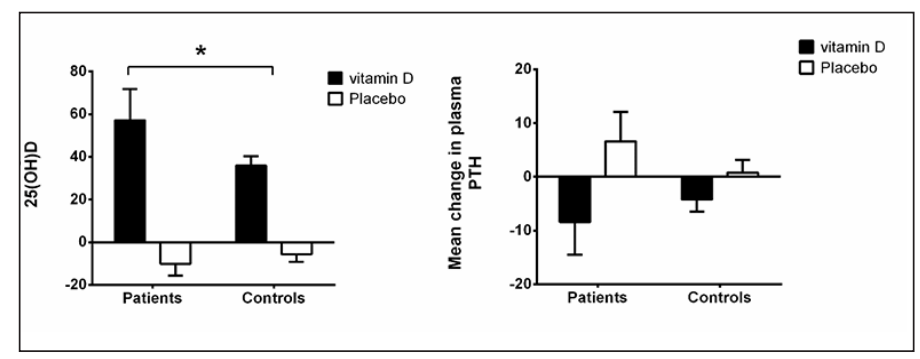

${ }^{*} \mathrm{p}<0.05$ by one-tail t-test

\section{Measures of degree of depression}

Baseline MDI total score was higher among the patients compared to the healthy controls $(\mathrm{P}<0.01$, and remained different at 6 and 12 months follow-up, by ANOVA $(\mathrm{P}<0.01$, Fig. $3 \mathrm{~A})$. Among the controls, a significant lower MDI score was rated among the vitamin $\mathrm{D}$ treated compared to placebo treated at six months follow-up ( $\mathrm{p}=0.01$ by $\mathrm{t}$-test for two samples), which became nonsignificant again at 12 months $(\mathrm{p}=0.15$ by t-test for two samples).
Figure 3

MDI score over time in patients and controls by treatment (vitamin D vs. placebo)

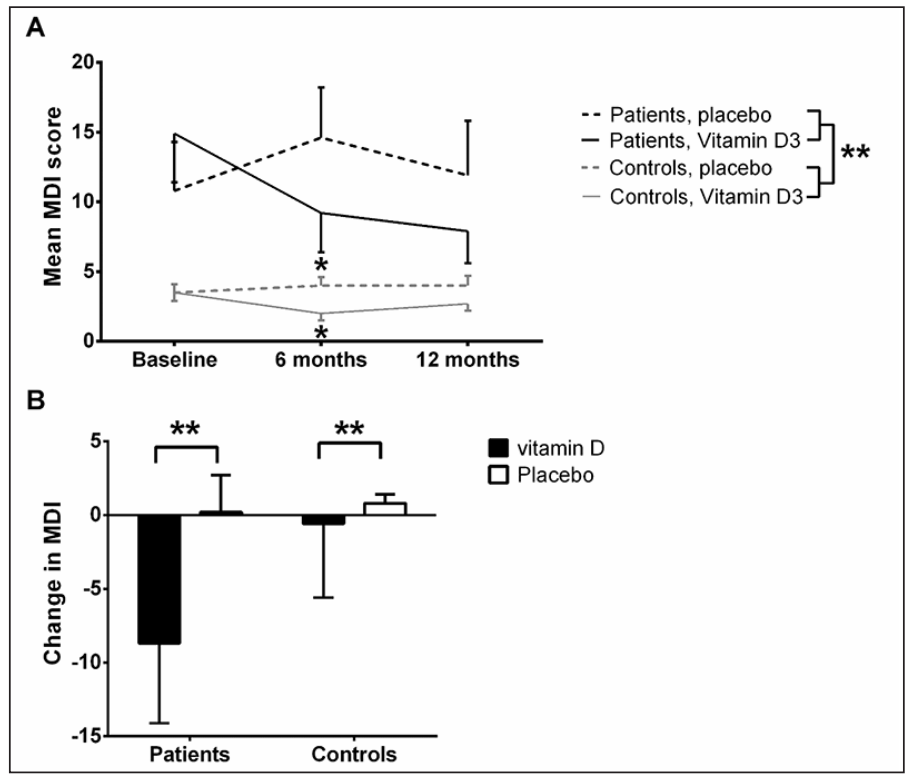

${ }^{*} \mathrm{P}<0.05,{ }^{* *} \mathrm{P}<0.01$; A: Absolute MDI total score over time (lower MDI total score is better self-perceived mental health). By ANOVA, no difference between vitamin $\mathrm{D}$ and placebo treated $(\mathrm{p}=0.39)$ was present, whereas a significant difference was present between patients and controls $(\mathrm{p}<0.01)$; B: Change in MDI depression score (delta MDI total score) from baseline to one year stratified by citalopram treated and controls randomised to vitamin D or placebo. Mean and SEM for changes.

An analysis of the changes in total MDI score over 12 month (delta MDI total score) revealed an improvement among vitamin $\mathrm{D}$ treated patients after 12 months as compared to placebo treated patients (mean improvement in MDI score when treated with vitamin $\mathrm{D}$ was $-8.7 \pm 5.4$ vs. a worsening with placebo of $0.2 \pm 2.5$ equalling a 
mean difference of $8.9 \pm 2.7, \mathrm{p}<0.01$ by standard normal distribution) (Fig. 3B). A difference was also present for the controls (difference in MDI score: $-0.6 \pm 0.5$ vs. $0.8 \pm 0.6$ equalling a mean difference of $1.4 \pm 0.3, \mathrm{p}<0.01$ by standard normal distribution - Fig. 3B). The symptoms that tended to improve were primarily the more severe, including all three core-symptoms of depression: feeling sad and sorry, lack of interest in daily activities, lack of energy, and the associated symptoms: decreased self-confidence, bad conscience, feeling restless, and to some degree that life was not worth living, difficulties concentrating, and decreased appetite (data not shown).

Overall, for patients and controls a positive correlation was present between changes in plasma $25(\mathrm{OH}) \mathrm{D}$ and changes in MDI total scores after 12 months of vitamin D treatment, with higher plasma 25(OH)D correlated to lower MDI scores (i.e. fewer depressive symptoms). This was significant among controls (Pearson's $r=0 .-43$, $\mathrm{p}<0.01$ ), but not among the citalopram treated.

\section{Handgrip strength}

Fig. 4 shows that after 12 month handgrip strength was significantly higher among the patients receiving vitamin $\mathrm{D}$ supplement compared to the patients receiving placebo ( 31.1 vs. $25.2 \mathrm{~kg}, \mathrm{P}=0.03$ ). Among the controls, no differences were present after 6 and 12 months. There were no correlations between the improvements in MDI and handgrip strength.

\section{Figure 4}

Handgrip strength. Values are mean and SEM. Dashed line is placebo, continuous line is vitamin D. Black line is controls, grey line are patients

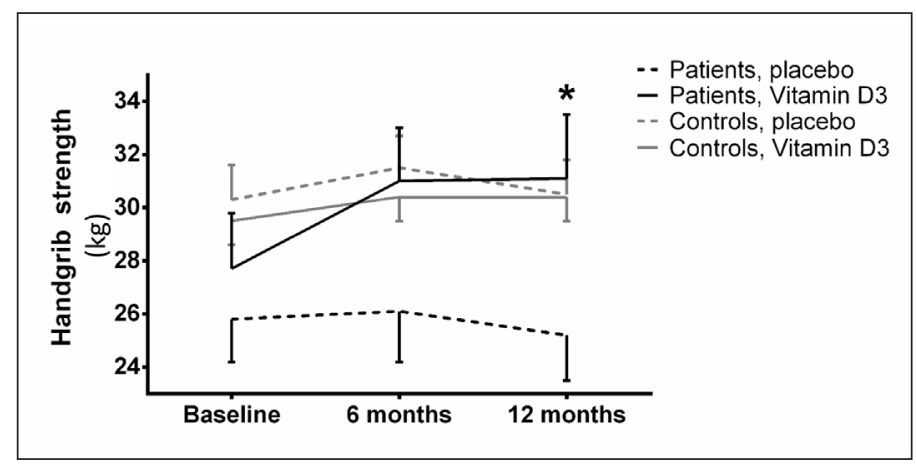

*: $\mathrm{p}<0.05$, when comparing vitamin D with placebo treatment at 12 months

\section{Timed Up and Go}

At baseline the TUG performance was significantly inferior (i.e. longer time needed to complete the test) among the patients when compared to controls ( $\mathrm{P}=0.01)$. After 6 and 12 months this difference became less pronounced $(\mathrm{P}=0.03$ and $\mathrm{P}=0.08)$.

\section{Postural control and clinical measures}

The changes in parameters of postural control as well as blood pressure and pulse did not differ between vitamin $\mathrm{D}$ and placebo treated neither among patients with major depressive disorder on citalopram nor among the healthy controls (data not shown). Body weight and height also remained unchanged between all groups.

\section{Pain measures}

At baseline patients experienced a higher level of self-perceived pain in daily life (higher EQ-5D pain rating) compared to controls (1.75 \pm 0.14 and $1.24 \pm 0.06$, respectively, $\mathrm{P}<0.01$ ) with no effect over time.

No significant effect of vitamin D3 treatment was present for cuff PDT and cuff PTT (t-tests, $p>0.05$ and ANOVA, $p>0.05$ - data not shown). Baseline TSP and $\mathrm{CPM}$ did not differ significantly when comparing patients and controls. No significant effect from 12-months vitamin D3 vs. placebo treatment could be detected on TSP and CPM (t-tests, $\mathrm{p}>0.05$ and ANOVA, $\mathrm{p}>0.05$ ).

\section{BMD and bone turnover markers}

The changes in BMD and bone turnover markers (TRAB5b and P1NP) did not differ between vitamin D and placebo treated neither among citalopram treated patients nor among the healthy controls.

\section{Discussion}

In this study, it was demonstrated shown that vitamin $\mathrm{D}$ may improve depressive symptoms in patients treated with citalopram, and improve handgrip strength.

\section{Vitamin D and depression}

The improvement in delta MDI total score among the patients treated with vitamin $\mathrm{D}$ after 12 months, suggest a potential beneficial effect that may be used as a simple and effective supplementary treatment for depression. This is supported by results from recent studies (11). The correlation between improvements in the MDI total score and increased plasma 25(OH)D for all subjects further indicates vitamin $\mathrm{D}$ as a likely contributor to improvement in mental health. This is in line with a large prospective cohort study of non-depressed women aged 55 to 69 years, who observed a significantly lower mental health-related quality of life when consuming $<400 \mathrm{IU} /$ day $[<10 \mu \mathrm{g} /$ day] of vitamin $\mathrm{D}$ compared to those who consumed $\geq 400 \mathrm{IU} /$ day (22).

The absolute decrease of around nine of 50 possible points in the individual depressive symptoms is a rather large absolute gain in well-being among the patients, and it may thus have a rather large clinical importance 
if confirmed in further studies. Furthermore, it is interesting, that the symptoms that tended to improve were primarily the more severe, including all three coresymptoms of depression: feeling sad and sorry, lack of interest in daily activities, lack of energy, and the associated symptoms: decreased self-confidence, bad conscience, feeling restless, and to some degree that life was not worth living, difficulties concentrating, and decreased appetite. Also among the controls, a trend of fewer depressive symptoms was seen among vitamin D treated compared to placebo.

\section{Potential cytochrome p450 (CYP)-enzyme interaction for citalopram and vitamin $D$}

The higher increase in serum 25(OH)D among citalopram treated patients compared to controls may be caused by an interaction between citalopram and the CYP system. Hence citalopram may occupy the CYP system preventing this from turning 25-OH Vitamin D into 24,25-OH-vitamin $\mathrm{D}$, instead facilitating the formation of 1,25-OH2-Vitamin D and thus a higher biological effect mirrored in the trend towards a larger decrease in PTH. Citalopram is metabolised by the CYP2C19 system, the inactive 25,25 -dihydroxy vitamin $\mathrm{D}$ is metabolised by the CYP24A1 enzyme, and 1,25-dihydroxy vitamin D is metabolised by the CYP27B1, while 25-hydroxy-vitamin $\mathrm{D}$ is metabolised from cholecalciferol by CYP27A1 [23]. Cholecalciferol may interact with CYP2C19 [24], and citalopram may thus potentially interact with cholecalciferol metabolism. Both CYP27B1 and CYP24A1 are expressed in the brain tissue suggesting that there may exist both synthesis and elimination, respectively, of vitamin D3 in the brain (25).

Voshaar et al. (26) investigated serum vitamin D levels among depressed elderly patients treated with antidepressants $(n=355)$ compared to non-depressed control subjects $(n=124)$. In contrast to the present study, they reported that TCA usage correlated with low $1,25-(\mathrm{OH}) 2$ vitamin D3 levels, but not $25(\mathrm{OH}) \mathrm{D}$, and neither SSRI nor newer types of antidepressants did effect any of those two compounds (26). This may be explained by possible different interactions between CYP enzymes and antidepressants during low basal 25(OH) D levels as compared to higher $25(\mathrm{OH}) \mathrm{D}$ levels induced by cholecalciferol supplements. It should be noted that baseline 25(OH)D levels was relatively higher among citalopram treated patients of the present study, than that reported in the study by Voshaar and colleagues (26).

\section{Grip strength, effect from vitamin D supplementation}

Despite the fact that patients of the present study were vitamin $\mathrm{D}$ replete (mean $25(\mathrm{OH}) \mathrm{D} \geq 86 \mathrm{nmol} / \mathrm{L}$ ) at baseline, an improvement in handgrip strength was found among patients receiving 2000 IU vitamin D supplement during the one year follow-up period compared to the placebo group. Actually - despite being older and thus having a priori lower hand grip strength - the patients on citalopram improved their hand grip strength to that of the controls. Currently, the effect of Vitamin D on muscle strength is controversial. A recent study report a decrease in hand grip strength in elderly vitamin D-sufficient women following vitamin D supplementation (27). However, this study (27) reports a relatively short follow up of three months whereas the present study has a longer follow up, which may suggest a dynamic in the effect of Vitamin D; vitamin D at steady state may improve muscle strength. However, short-term supplementation with no steady state may decrease muscle strength. Improved well-being from diminished depressive symptoms may potentially lead to increased physical activity and thus increased strength. However, no correlations were present in our study between improvements in MDI and handgrip strength.

\section{Vitamin D, pain and pain sensitivity}

The patients had higher levels of self-perceived pain at baseline compared to controls. Despite this, no differences were found in pain sensitivity measures between patients and controls and no changes were observed following treatment.

High clinical pain ratings and long painful durations (years) have been found associated with increased pain sensitivity and this has been documented in multiple painful conditions (28). Pain sensitivity is influenced by multiple factors and cognitive factors and pain sensitivity have been found associated in recent studies (28). Duloxetine (an antidepressant) has demonstrated analgesic effects in chronic pain patients (28) and has been suggested to target the pain inhibitory pathways (10) suggesting a link between depression and pain sensitivity. Despite this, the clinical pain ratings in the current study were low, which could explain why patients treated with anti-depressants and controls were not found different in regards to the pain sensitivity measures. The vitamin D-induced analgesic effect of antidepressants, may be an involvement of vitamin D in neuronal functioning through production and/or regulation of various monoamines including serotonin and noradrenaline (29) and neurotrophic factors with the potential to alter pain sensitivity. Serotonin and noradrenaline are mainly described to be involved in pain inhibitory pathways (30) but the current study was unable to demonstrate a significant change in pain inhibitory pathways after treatment, which might be due to the lack of impairment in the pain inhibitory pathways at baseline. 


\section{Strengths and limitations}

The main advantage of the present randomized double-blinded placebo controlled study was the homogeneous group of subjects using only one type of antidepressant (citalopram), i.e. confounding from multiple drugs was avoided. Furthermore, a standardized dose of vitamin $\mathrm{D}$ that induced a significant increase in serum vitamin D was used, which was raised from within the normal level to the upper range of the normal level, i.e. it was possible to study the effects of high levels of vitamin $\mathrm{D}$, and also at these levels no effects were seen.

The main limitation of our study was the low number of patients in the patient group and that no males were studied.

\section{Conclusion}

In conclusion, vitamin D may improve depressive symptoms in patients treated with citalopram, and potentially improve handgrip strength. However, further research on larger cohorts is needed to clarify the clinical implications.

Funding: Peter Vestergaard reports grants from The Obel Family Foundation (\#25243), grants from The AP Møller and Chastine Maersk Mc. Kinny Møller Foundation (\#01) during the conduct of the study; Thomas Graven-Nielsen and Kristian Kjær Petersen reports grants from The Danish National Research Foundation (\#DNRF121), during the conduct of the study. The sponsors had no role in the design and conduct of the study; in the collection, analysis, and interpretation of data; in the preparation of the manuscript; or in the review or approval of the manuscript. Stine Aistrup Eriksen, Jakob Starup-Linde and Rogerio Pessoto Hirata has nothing to disclose. Therefore all authors: Stine Aistrup Eriksen, Jakob Starup-Linde, Rogerio Pessoto Hirata, Kristian Kjær Petersen, Thomas Graven-Nielsen and Peter Vestergaard report no conflicts of interests.

Acknowledgements: The Obel Family Foundation and the AP Møller and Chastine Maersk Mc. Kinny Møller Foundation are acknowledged for providing financial support. Center for Neuroplasticity and Pain (CNAP) is supported by the Danish National Research Foundation. The management and staff at Center for Clinical and Basic Research (CCBR) Aalborg, are acknowledged for providing optimal clinical facilities and professional execution of clinical measures (DXA and blood sampling), as well as continuous support and guidance in good clinical practice.

Conflict of interest: No conflicts of interests.

Ethical standard: The study followed the Helsinki declaration and all participants gave informed written consent.

\section{References}

1. H. A. Bischoff-Ferrari m.fl., "Effect of Vitamin D on falls: a meta-analysis", JAMA, bd. 291, nr. 16, s. 1999-2006, apr. 2004.

2. L. Ceglia og S. S. Harris, "Vitamin D and its role in skeletal muscle", Calcif. Tissue Int., bd. 92, nr. 2, s. 151-162, feb. 2013.

3. C. Annweiler, A. M. Schott, G. Berrut, B. Fantino, og O. Beauchet, "Vitamin D-related changes in physical performance: a systematic review", J Nutr Health Aging, bd. 13, nr. 10, s. 893-898, dec. 2009.

4. S. W. Muir og M. Montero-Odasso, "Effect of vitamin D supplementation on muscle strength, gait and balance in older adults: a systematic review and meta-analysis", J Am Geriatr Soc, bd. 59, nr. 12, s. 2291-2300, dec. 2011.

5. B. Stubbs, L. Eggermont, S. Patchay, og P. Schofield, "Older adults with chronic musculoskeletal pain are at increased risk of recurrent falls and the brief pain inventory could help identify those most at risk", Geriatr Geronto Int, bd. 15, nr. 7, s. 881-888, jul. 2015.

6. A. Fekadu, L. J. Rane, S. C. Wooderson, K. Markopoulou, L. Poon, og A. J. Cleare, "Prediction of longer-term outcome of treatment-resistant depression in tertiary care.", The British journal of psychiatry : the journal of mental science, bd. 201, s. 369-75, nov. 2012.

7. R. E. S. Anglin, Z. Samaan, S. D. Walter, og S. D. McDonald, "Vitamin D deficiency and depression in adults: systematic review and meta-analysis.", The British journal of psychiatry : the journal of mental science, bd. 202, nr. 2, s. $100-7$, feb. 2013.

8. E. W. de Heer m.fl., "The association of depression and anxiety with pain: a study from NESDA", PLoS ONE, bd. 9, nr. 10, s. e106907, 2014.

9. J. F. Karp, J. Scott, P. Houck, C. F. Reynolds, D. J. Kupfer, og E. Frank, “Pain predicts longer time to remission during treatment of recurrent depression", J Clin Psychiatry, bd. 66, nr. 5, s. 591-597, maj 2005

10. D. Yarnitsky, M. Granot, H. Nahman-Averbuch, M. Khamaisi, og Y. Granovsky, "Conditioned pain modulation predicts duloxetine efficacy in painful diabetic neuropathy", Pain, bd. 153, nr. 6, s. 1193-1198, jun. 2012.

11. J. Sarris m.fl., "Adjunctive Nutraceuticals for Depression: A Systematic Review and Meta-Analyses", American Journal of Psychiatry, bd. 173, nr. 6, s. 575-587, jun. 2016.

12. E. Garcion, N. Wion-Barbot, C. N. Montero-Menei, F. Berger, og D. Wion, "New clues about vitamin D functions in the nervous system.", Trends in endocrinology and metabolism: TEM, bd. 13, nr. 3, s. 100-5, apr. 2002.

13. R. von Känel, V. Müller-Hartmannsgruber, G. Kokinogenis, og N. Egloff, "Vitamin D and central hypersensitivity in patients with chronic pain", Pain Med, bd. 15, nr. 9, s. 1609-1618, sep. 2014.

14. M. Gaikwad, S. Vanlint, M. Mittinity, G. L. Moseley, og N. Stocks, “Does vitamin D supplementation alleviate chronic nonspecific musculoskeletal pain? A systematic review and meta-analysis", Clin. Rheumatol., bd. 36, nr. 5, s. 1201-1208, maj 2017.

15. Z. Wu, Z. Malihi, A. W. Stewart, C. M. Lawes, og R. Scragg, "Effect of Vitamin D Supplementation on Pain: A Systematic Review and Metaanalysis", Pain Physician, bd. 19, nr. 7, s. 415-427, okt. 2016.

16. S. Schwan og P. Hallberg, "SSRIs, bone mineral density, and risk of fractures-a review", Eur Neuropsychopharmacol, bd. 19, nr. 10, s. 683-692, okt. 2009.

17. P. Vestergaard, D. Prieto-Alhambra, M. K. Javaid, og C. Cooper, "Fractures in users of antidepressants and anxiolytics and sedatives: effects of age and dose", Osteoporos Int, bd. 24, nr. 2, s. 671-680, feb. 2013.

18. L. R. Olsen, D. V. Jensen, V. Noerholm, K. Martiny, og P. Bech, “The internal and external validity of the Major Depression Inventory in measuring severity of depressive states", Psychol Med, bd. 33, nr. 2, s. 351-356, feb. 2003.

19. S. J. Coons, S. Rao, D. L. Keininger, og R. D. Hays, “A comparative review of generic quality-of-life instruments", Pharmacoeconomics, bd. 17, nr. 1, s. 13-35, jan. 2000.

20. T. Graven-Nielsen, H. B. Vaegter, S. Finocchietti, G. Handberg, og L. ArendtNielsen, "Assessment of musculoskeletal pain sensitivity and temporal summation by cuff pressure algometry: a reliability study", Pain, bd. 156, nr. 11, s. 2193-2202, nov. 2015.

21. K. K. Petersen, T. Graven-Nielsen, O. Simonsen, M. B. Laursen, og L. Arendt-Nielsen, "Preoperative pain mechanisms assessed by cuff algometry are associated with chronic postoperative pain relief after total knee replacement", Pain, bd. 157, nr. 7, s. 1400-1406, jul. 2016.

22. S. Motsinger, D. Lazovich, R. F. MacLehose, C. J. Torkelson, og K. Robien, "Vitamin D intake and mental health-related quality of life in older women: the Iowa Women's Health Study", Maturitas, bd. 71, nr. 3, s. 267-273, mar. 2012.

23. D. E. Prosser og G. Jones, "Enzymes involved in the activation and inactivation of vitamin D", Trends Biochem. Sci., bd. 29, nr. 12, s. 664-673, dec. 2004.

24. H. Yamazaki og T. Shimada, "Effects of arachidonic acid, prostaglandins, retinol, retinoic acid and cholecalciferol on xenobiotic oxidations catalysed by human cytochrome P450 enzymes", Xenobiotica, bd. 29, nr. 3, s. 231-241, mar. 1999.

25. L. R. Harms, T. H. J. Burne, D. W. Eyles, og J. J. McGrath, “Vitamin D and the brain", Best Pract. Res. Clin. Endocrinol. Metab., bd. 25, nr. 4, s. 657-669, aug 2011.

26. R. C. Oude Voshaar, W. J. Derks, H. C. Comijs, R. A. Schoevers, M. H. de Borst, og R. M. Marijnissen, "Antidepressants differentially related to 1,25$(\mathrm{OH})_{2}$ vitamin $\mathrm{D}_{3}$ and $25-(\mathrm{OH})$ vitamin $\mathrm{D}_{3}$ in late-life depression", Trans Psychiatry, bd. 4, s. e383, apr. 2014.

27. L. S. Bislev, L. Langagergaard Rødbro, L. Rolighed, T. Sikjaer, og L. Rejnmark, "Effects of Vitamin D3 Supplementation on Muscle Strength, Mass, and Physical Performance in Women with Vitamin D Insufficiency: A Randomized Placebo-Controlled Trial", Calcif. Tissue Int., jun. 2018.

28. L. Arendt-Nielsen m.fl., "Assessment and manifestation of central sensitisation across different chronic pain conditions", Eur J Pain, bd. 22, nr. 2, s. 216-241, 2018.

29. E. A. Shipton og E. E. Shipton, "Vitamin D and Pain: Vitamin D and Its Role in the Aetiology and Maintenance of Chronic Pain States and Associated Comorbidities", Pain Res Treat, bd. 2015, s. 904967, 2015.

30. K. Bannister og A. H. Dickenson, "What the brain tells the spinal cord", Pain, bd. 157, nr. 10, s. 2148-2151, 2016 